\title{
Application of spermidine to manage water stress for improved fine rice yield and quality
}

\author{
Naeem Sarwar ${ }^{*}$, Muhammad Haseeb Javaid ${ }^{1}$, Asifa Neelam ${ }^{1}$, Atique- \\ ur-Rehaman ${ }^{1}$, Omer Farooq ${ }^{1}$, Allah Wasaya ${ }^{2}$, Khuram Mubeen ${ }^{3}$, Aamir \\ Ghani $^{4}$ and Muhammad Zaighum Mushtaq ${ }^{5}$ \\ 1. Department of Agronomy, Bahauddin Zakariya University Multan-Pakistan \\ 2. College of Agriculture, Bahauddin Zakariya University, Bahadur Sub-Campus Layyah-Pakistan \\ 3. Department of Agronomy, Muhammad Nawaz Sharif University of Agriculture Multan-Pakistan \\ 4. Maize and Millets Research Institute Yusafwala, Sahiwal-Pakistan \\ 5. Biochemistry section Post Harvest Research Centre Faisalabad-Pakistan \\ *Corresponding author's email: naeemsarwar@bzu.edu.pk \\ Citation \\ Naeem Sarwar, Muhammad Haseeb Javaid, Asifa Neelam, Atique-ur-Rehaman, Omar Farooq, Allah Wasaya, \\ Khuram Mubeen, Aamir Ghani and Muhammad Zaighum Mushtaq. Application of spermidine to manage water \\ stress for improved fine rice yield and quality. Pure and Applied Biology. Vol. 9, Issue 3, pp1813-1819. \\ http://dx.doi.org/10.19045/bspab.2020.90193
}

Received: 05/02/2020 Revised: $25 / 04 / 2020$ Accepted: $29 / 04 / 2020$

Online First: 11/05/2020

\section{Abstract}

Climate change has created challenges for the rice producers and farmers are shifting rice cultivation in aerobic system to save irrigation water. Although in aerobic rice, crop can be matured with less irrigation water but farmers have to compromise yield reduction linear to water saving. Spermidin is plant hormone which has significant role to stand plant in water stress condition. So, in this field experiment, we foliarly applied spermidine at seedling $\left(\mathrm{F}_{1}\right)$, tillering $\left(\mathrm{F}_{2}\right)$, panicle intiation $\left(\mathrm{F}_{3}\right)$, seedling and panicle initiation $\left(\mathrm{F}_{4}\right)$, tillering and panicle initiation $\left(\mathrm{F}_{5}\right)$, Seedling, Tillering and Panicle initiation $\left(\mathrm{F}_{6}\right)$ under aerobic and flooded condition. We observed different growth, yield and quality parameters during course of study. Spermidin application resulted in improved crop performance both in aerobic as well as in flooded rice. Treatments comparison revealed that spermidin application at panicle initiation stage resulted in better crop performance in sense of economics involved. Furthermore, spermidine application also improved rice quality which is one of most important factor in fine rice. In a nutshell, it can be suggested that aerobic rice productivity can be improved with spermidin application at panicle initiation stage to couple water stress.

Keywords: Growth; Grain quality; Rice; Spermidin: Yield

\section{Introduction}

To subsidize farmers, scientists are working on an innovative way of growing rice in aerobic conditions. This technique does not require standing water conditions or transplantation of nursery. However, the productivity of rice grown under non-flooded conditions leads to lower grain yield and poor quality [1]. The stakeholders observed that rice grown under aerobic conditions demonstrate less yield of about 2 t/ha comparative to 3-3.5 t/ha under flooded 
conditions [2]. For successful aerobic rice production, various growth regulators are being used like auxin, gibberellins and polyamines [3].

Polyamines comprise of three types, namely spermidine, spermine, and putrescine. Among all three types; spermidine is known to have multiple functions in plant growth and development $[4,5]$. Studies exhibited that it has an appreciable role to cope with stress situations in plants [5]. Various physiological, biological and metabolic processes are regulated by spermidine. It improves transcription, modification in RNA or DNA and cell growth [6], modulate enzymatic and protein synthetic activities [7, 8] when accumulating in high concentration. [9] reported that plants having a high concentration of spermidine establish high yield and yield components. In stress conditions, they also have antioxidative properties. It has the ability to behave like scavenger or antioxidant enzymes for free radicals to mitigate oxidative injury $[10,11]$. To see the trend of aerobic rice production and compromising of promising yield by farmers to save water, we hypothysed that application of spermidin in aerobic rice at most important stage may combat water stress to improve crop yield and quality.

\section{Materials and methods}

Experiment was carried out at Agronomic Research Farm, Bahauddin Zakariya University Multan. There were two factors: cultivation systems i.e. Aerobic rice $\left(\mathrm{T}_{1}\right)$ and Transplanted flooded rice $\left(\mathrm{T}_{2}\right)$. In second factor, spermidin was applied at different growth stages i.e at seedling, tillering, panicle initiation alone or in combination of each other along with one treatment in which spermidin was applied at all three stages. All treatments were arranged under split plot, having cultivation systems in main plot and spermidin application in sub-plots. Seed of fine rice cultivar: Super Basmati was used as test cultivar. Firstly, field was selected for the experiment and rauni irrigation was provided to improve soil moisture as well as for weed emergence. After weed emergence, field was cultivated and prepared for nursery as well as for sowing of aerobic rice. Aerobic rice was direct seeded in the field while at the same date we sowed nursery for transplanted rice. Seed rate of $15 \mathrm{~kg} / \mathrm{acre}$ was used for aerobic rice with $\mathrm{R} \times \mathrm{R}$ distance of $25 \mathrm{~cm}$. Nursery was raised by following wet seeded method, in which, farm yard manure was mixed in the soil before sowing and then sprouted seeded was broadcasted. Seed was spreaded at $2 \mathrm{~kg} / \mathrm{marla}$ and then irrigation was provided as per crop need. After 25 days, nursery was transplanted in the main field at distance of $25 \mathrm{~cm} \mathrm{R} \mathrm{x} \mathrm{R} \mathrm{and} \mathrm{P} \mathrm{x} \mathrm{P.} \mathrm{Spermidin}$ solution was prepared in lab with the use of distilled water and was applied as per treatment.

The sample was obtained fortnightly after a month of the plantation to calculate leaf area index using leaf area meter. All growth parameters such as leaf area duration (LAD), leaf area index (LAI), crop growth rate (CGR), net assimilation rate (NAR) was calculated. When the crop reached physiological maturity, it was manually harvested and various parameters were noted Results

\section{Growth analysis}

Leaf area index was increasing from emergence to panicle initiation as shown in (Fig. 1) after then it starts decreasing till maturity. Crop significantly improved leaf area index in flooded condition as well as with spermidin application. Treatment combination of $\mathrm{T}_{2} \mathrm{~S}_{7}$, resulted in highest level followed by treatments $T_{2} S_{6}, T_{2} S_{5}$ and $T_{1} S_{7}$. Almost similar results were found in LAD, CGR and NAR, where three time application of spermidin under flooded condition was found supreme. This incremental effect of spermidin application was found in line with flooded condition and $\mathrm{S}_{7}$ treatment enhanced crop growth (Table 1). 


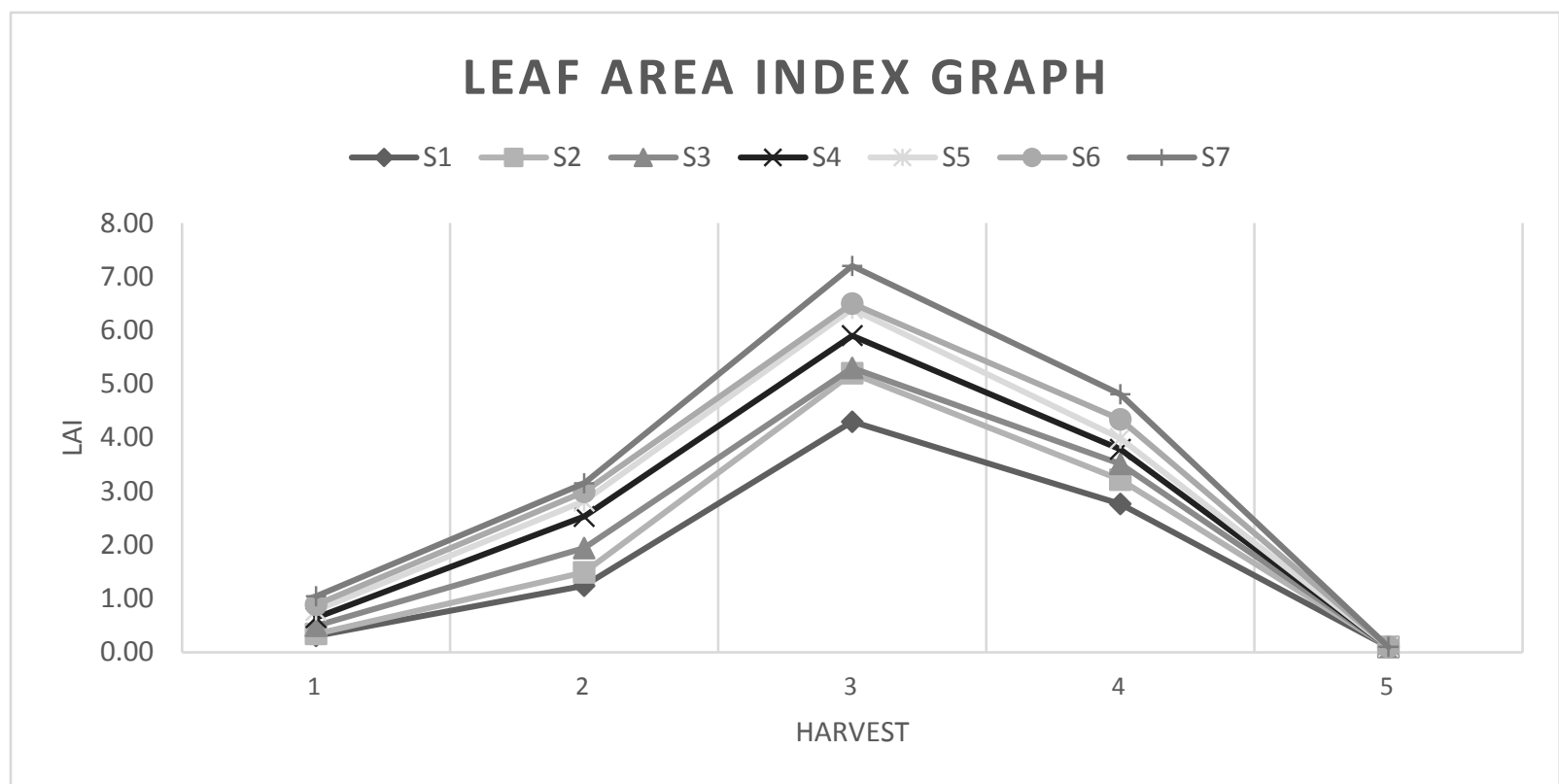

Figure 1. Effect of spermidine application on Leaf area Index (LAI) of rice crop at different harvest

Table 1. Effect of spermidine application on growth parameters of rice crop at different developmental stages

\begin{tabular}{|c|c|c|c|c|}
\hline Treatments & LAI & LAD (days) & CGR $\left(\mathrm{gm}^{-2} \mathrm{day}^{-1}\right)$ & NAR $\left(\mathrm{gm}^{-2} \mathrm{day}^{-1}\right)$ \\
\hline $\mathbf{T}_{1} \mathbf{S}_{1}$ & $3.93 \mathrm{~g}$ & $92.56 \mathrm{~h}$ & $10.45 \mathrm{~h}$ & $3.60 \mathrm{~g}$ \\
\hline $\mathbf{T}_{1} \mathbf{S}_{2}$ & $4.96 \mathrm{e}$ & $113.05 \mathrm{~g}$ & $12.94 \mathrm{fg}$ & $4.47 \mathrm{f}$ \\
\hline $\mathbf{T}_{1} \mathbf{S}_{3}$ & $4.96 \mathrm{e}$ & $114.36 \mathrm{~g}$ & $13.47 \mathrm{f}$ & $4.56 \mathrm{f}$ \\
\hline $\mathbf{T}_{1} \mathbf{S}_{4}$ & $5.70 \mathrm{~d}$ & $132.40 \mathrm{f}$ & $14.65 \mathrm{e}$ & $5.88 \mathrm{e}$ \\
\hline $\mathbf{T}_{1} \mathbf{S}_{5}$ & $6.16 \mathrm{c}$ & $147.40 \mathrm{e}$ & $16.79 \mathrm{c}$ & $7.32 \mathrm{~d}$ \\
\hline $\mathbf{T}_{1} \mathbf{S}_{6}$ & $6.26 \mathrm{c}$ & $152.66 \mathrm{de}$ & $17.01 \mathrm{c}$ & $7.92 \mathrm{c}$ \\
\hline $\mathbf{T}_{1} \mathbf{S}_{7}$ & $6.86 \mathrm{~b}$ & $174.20 \mathrm{~b}$ & $18.35 \mathrm{~b}$ & $9.16 \mathrm{~b}$ \\
\hline $\mathbf{T}_{2} \mathbf{S}_{1}$ & $4.60 \mathrm{f}$ & $106.03 \mathrm{~g}$ & $12.16 \mathrm{~g}$ & $4.60 \mathrm{f}$ \\
\hline $\mathbf{T}_{2} \mathbf{S}_{2}$ & $5.43 \mathrm{~d}$ & $128.70 \mathrm{f}$ & $14.99 \mathrm{e}$ & $5.35 \mathrm{e}$ \\
\hline $\mathbf{T}_{2} \mathbf{S}_{3}$ & $5.53 \mathrm{~d}$ & $131.66 \mathrm{f}$ & $15.36 \mathrm{de}$ & $5.57 \mathrm{e}$ \\
\hline $\mathbf{T}_{2} \mathbf{S}_{4}$ & $6.13 \mathrm{c}$ & $143.40 \mathrm{e}$ & $16.29 \mathrm{~cd}$ & $6.91 \mathrm{~d}$ \\
\hline $\mathbf{T}_{2} \mathbf{S}_{5}$ & $6.60 \mathrm{~b}$ & $160.36 \mathrm{~cd}$ & $18.44 \mathrm{~b}$ & $8.69 \mathrm{c}$ \\
\hline $\mathbf{T}_{2} \mathbf{S}_{6}$ & $6.66 \mathrm{~b}$ & $163.06 \mathrm{c}$ & $18.55 \mathrm{~b}$ & $9.15 \mathrm{~b}$ \\
\hline $\mathbf{T}_{2} \mathbf{S}_{7}$ & $7.50 \mathrm{a}$ & $186.06 \mathrm{a}$ & $19.82 \mathrm{a}$ & $9.87 \mathrm{a}$ \\
\hline LSD & 0.30 & 9.36 & 0.91 & 0.70 \\
\hline
\end{tabular}

\section{Yield attributes}

Crop significantly improved yield attributes with spermidin application in both cultivation systems. Taller plants were observed in treatment $\mathrm{T}_{2} \mathrm{~S}_{7}$ where three time spermidin was applied in transplanted rice which was followed by $T_{2} S_{6}, T_{2} S_{5}$. This incremental effect was also observed in aerobic rice. Treatment combination of $\mathrm{T}_{1} \mathrm{~S}_{1}$ showed shorter plants. In case of panicle length, longer panicles were recorded in treatments $\mathrm{T}_{2} \mathrm{~S}_{7}, \mathrm{~T}_{2} \mathrm{~S}_{6}, \mathrm{~T}_{2} \mathrm{~S}_{5}, \mathrm{~T}_{2} \mathrm{~S}_{4}$ under flooded rice which were similar with $\mathrm{T}_{1} \mathrm{~S}_{7}$ of aerobic rice. This trend was also very similar to grain 
weight, as heavier grains were recorded in $_{2} \mathrm{~S}_{7}$ which was similar with $\mathrm{T}_{1} \mathrm{~S}_{7}$. Flooded rice also resulted in higher biological yield in treatments $\mathrm{T}_{2} \mathrm{~S}_{7}, \mathrm{~T}_{2} \mathrm{~S}_{6}, \mathrm{~T}_{2} \mathrm{~S}_{5}$ while lowest biological yield was recorded in $\mathrm{T}_{1} \mathrm{~S}_{1}$ where spermidin application was missed. In case of grain yield, treatment combination of $\mathrm{T}_{2} \mathrm{~S}_{7}$ was found superior followed by $T_{2} S_{6}, T_{2} S_{5}$ in flooded rice and $T_{1} S_{7}$ in aerobic rice which explained that performance of aerobic rice was very close to flooded rice under spermidin application (Table 2).

\section{Grain quality}

Kernels having specific characteristics for quality concern was counted to see the impact of spermide on rice grain quality. Trend showed that normel kernels were highest in treatments combination of $\mathrm{T}_{2} \mathrm{~S}_{7}, \mathrm{~T}_{2} \mathrm{~S}_{6}, \mathrm{~T}_{2} \mathrm{~S}_{4}$ in flooded rice and $\mathrm{T}_{1} \mathrm{~S}_{7}, \mathrm{~T}_{1} \mathrm{~S}_{6}, \mathrm{~T}_{1} \mathrm{~S}_{5}$ in aerobic rice. Spermidin missing treatment resulted in lowest normal kernel in flooded as well as in aerobic rice. Trend of problematic kernels in term of abortive, sterile and opaque represented that spermidin application significantly reduced these kernels. Lowest kernels were appearing in treatment $\mathrm{T}_{2} \mathrm{~S}_{7}$ and $\mathrm{T}_{1} \mathrm{~S}_{7}$ in which three time application of spermidin was done. Highest unwanted kernels were appear in control treatment in which spermidin application was avoided (Table 3).

\section{Economic analysis}

Economic is the major parameter on which adoptability of any technique is dependent and is most crucial in developing countries. Aerobic rice found to be superior on flooded rice when compared with benefit cost ratio. Economical comparison of different treatment revealed that maximum $\mathrm{BCR}$ was achieved in $T_{1} S_{7}$, followed by $T_{1} S_{4}, T_{2} S_{7}$, $\mathrm{T}_{2} \mathrm{~S}_{4}$. Results explained that aerobic rice can improve productivity and enhanced economic return with the application of spermidin at three different developmental stages (T1S7) or two stages (seedling + panicle initiation) (Table 4).

Table 2. Response of yield attributes to spermidine application

\begin{tabular}{|c|c|c|c|c|c|}
\hline Treatments & $\begin{array}{c}\text { Plant height } \\
\text { (cm) }\end{array}$ & $\begin{array}{c}\text { Panicle } \\
\text { length }(\mathbf{c m})\end{array}$ & $\begin{array}{c}\text { 1000-grain } \\
\text { weight }(\mathbf{g})\end{array}$ & $\begin{array}{c}\text { Biological yield } \\
\text { (kg/ha) }\end{array}$ & $\begin{array}{c}\text { Grain yield } \\
\text { (kg/ha) }\end{array}$ \\
\hline $\mathbf{T}_{\mathbf{1}} \mathbf{S}_{\mathbf{1}}$ & $91.66 \mathrm{k}$ & $21.33 \mathrm{f}$ & $19.80 \mathrm{~h}$ & $4512.47 \mathrm{f}$ & $2890.33 \mathrm{~h}$ \\
\hline $\mathbf{T}_{\mathbf{1}} \mathbf{S}_{\mathbf{2}}$ & $100.66 \mathrm{i}$ & $23.66 \mathrm{e}$ & $20.50 \mathrm{~g}$ & $6048.08 \mathrm{~d}$ & $3950.33 \mathrm{~g}$ \\
\hline $\mathbf{T}_{\mathbf{1}} \mathbf{S}_{\mathbf{3}}$ & $106.33 \mathrm{~g}$ & $23.66 \mathrm{e}$ & $20.87 \mathrm{~g}$ & $6098.39 \mathrm{~d}$ & $3988.00 \mathrm{~g}$ \\
\hline $\mathbf{T}_{\mathbf{1}} \mathbf{S}_{\mathbf{4}}$ & $108.33 \mathrm{f}$ & $26.00 \mathrm{~cd}$ & $30.91 \mathrm{c}$ & $6115.31 \mathrm{~d}$ & $4663.33 \mathrm{~d}$ \\
\hline $\mathbf{T}_{\mathbf{1}} \mathbf{S}_{\mathbf{5}}$ & $116.00 \mathrm{c}$ & $26.33 \mathrm{bc}$ & $29.20 \mathrm{~d}$ & $6768.21 \mathrm{bc}$ & $4831.33 \mathrm{~d}$ \\
\hline $\mathbf{T}_{\mathbf{1}} \mathbf{S}_{\mathbf{6}}$ & $116.33 \mathrm{c}$ & $26.33 \mathrm{bc}$ & $29.69 \mathrm{~d}$ & $6846.21 \mathrm{~b}$ & $5165.66 \mathrm{c}$ \\
\hline $\mathbf{T}_{\mathbf{1}} \mathbf{S}_{\mathbf{7}}$ & $117.66 \mathrm{c}$ & $27.00 \mathrm{ab}$ & $35.45 \mathrm{a}$ & $7442.92 \mathrm{~b}$ & $5720.66 \mathrm{~b}$ \\
\hline $\mathbf{T}_{\mathbf{2}} \mathbf{S}_{\mathbf{1}}$ & $97.66 \mathrm{j}$ & $22.00 \mathrm{f}$ & $24.10 \mathrm{f}$ & $4946.78 \mathrm{e}$ & $3900.33 \mathrm{~g}$ \\
\hline $\mathbf{T}_{\mathbf{2}} \mathbf{S}_{\mathbf{2}}$ & $103.33 \mathrm{~h}$ & $25.00 \mathrm{~d}$ & $24.91 \mathrm{e}$ & $6566.68 \mathrm{c}$ & $4277.33 \mathrm{f}$ \\
\hline $\mathbf{T}_{\mathbf{2}} \mathbf{S}_{\mathbf{3}}$ & $111.00 \mathrm{e}$ & $25.00 \mathrm{~d}$ & $25.27 \mathrm{e}$ & $6745.72 \mathrm{bc}$ & $4554.33 \mathrm{e}$ \\
\hline $\mathbf{T}_{\mathbf{2}} \mathbf{S}_{\mathbf{4}}$ & $113.00 \mathrm{~d}$ & $27.00 \mathrm{ab}$ & $33.71 \mathrm{~b}$ & $7189.96 \mathrm{~b}$ & $4886.33 \mathrm{~d}$ \\
\hline $\mathbf{T}_{\mathbf{2}} \mathbf{S}_{\mathbf{5}}$ & $124.33 \mathrm{~b}$ & $27.00 \mathrm{ab}$ & $33.25 \mathrm{~b}$ & $7897.91 \mathrm{ab}$ & $5461.33 \mathrm{~b}$ \\
\hline $\mathbf{T}_{\mathbf{2}} \mathbf{S}_{\mathbf{6}}$ & $125.66 \mathrm{~b}$ & $27.00 \mathrm{ab}$ & $33.50 \mathrm{~b}$ & $8349.29 \mathrm{a}$ & $5532.66 \mathrm{~b}$ \\
\hline $\mathbf{T}_{\mathbf{2}} \mathbf{S}_{\mathbf{7}}$ & $134.00 \mathrm{a}$ & $27.66 \mathrm{a}$ & $35.60 \mathrm{a}$ & $8876.04 \mathrm{a}$ & $6422.66 \mathrm{a}$ \\
\hline $\mathbf{L} \mathbf{S D}$ & 4.15 & 0.96 & 0.88 & 693.80 & 268.32 \\
\hline
\end{tabular}


Table 3. Rice quality trend effected with spermidine application

\begin{tabular}{|c|c|c|c|c|}
\hline Treatments & Normal Kernel (\%) & Abortive Kernel (\%) & $\begin{array}{c}\text { Sterile kernel } \\
(\boldsymbol{\%})\end{array}$ & $\begin{array}{c}\text { Opaque kernel } \\
(\%)\end{array}$ \\
\hline $\mathbf{T}_{\mathbf{1}} \mathbf{S}_{\mathbf{1}}$ & $53.35 \mathrm{f}$ & $10.19 \mathrm{a}$ & $12.66 \mathrm{a}$ & $26.28 \mathrm{a}$ \\
\hline $\mathbf{T}_{\mathbf{1}} \mathbf{S}_{\mathbf{2}}$ & $62.97 \mathrm{e}$ & $7.79 \mathrm{~b}$ & $9.55 \mathrm{~b}$ & $21.48 \mathrm{~b}$ \\
\hline $\mathbf{T}_{\mathbf{1}} \mathbf{S}_{\mathbf{3}}$ & $65.75 \mathrm{de}$ & $6.16 \mathrm{c}$ & $7.74 \mathrm{~cd}$ & $20.44 \mathrm{bc}$ \\
\hline $\mathbf{T}_{\mathbf{1}} \mathbf{S}_{\mathbf{4}}$ & $73.29 \mathrm{c}$ & $3.97 \mathrm{de}$ & $6.24 \mathrm{~d}$ & $18.00 \mathrm{de}$ \\
\hline $\mathbf{T}_{\mathbf{1}} \mathbf{S}_{\mathbf{5}}$ & $74.77 \mathrm{ab}$ & $3.84 \mathrm{de}$ & $5.27 \mathrm{de}$ & $16.50 \mathrm{ef}$ \\
\hline $\mathbf{T}_{\mathbf{1}} \mathbf{S}_{\mathbf{6}}$ & $75.06 \mathrm{ab}$ & $3.65 \mathrm{e}$ & $4.20 \mathrm{e}$ & $16.51 \mathrm{ef}$ \\
\hline $\mathbf{T}_{\mathbf{1}} \mathbf{S}_{\mathbf{7}}$ & $78.68 \mathrm{a}$ & $3.11 \mathrm{ef}$ & $3.81 \mathrm{f}$ & $15.33 \mathrm{gh}$ \\
\hline $\mathbf{T}_{\mathbf{2}} \mathbf{S}_{\mathbf{1}}$ & $54.29 \mathrm{f}$ & $9.44 \mathrm{a}$ & $9.91 \mathrm{~b}$ & $22.55 \mathrm{~b}$ \\
\hline $\mathbf{T}_{\mathbf{2}} \mathbf{S}_{\mathbf{2}}$ & $68.60 \mathrm{~d}$ & $5.95 \mathrm{c}$ & $7.77 \mathrm{c}$ & $18.88 \mathrm{~cd}$ \\
\hline $\mathbf{T}_{\mathbf{2}} \mathbf{S}_{\mathbf{3}}$ & $66.05 \mathrm{de}$ & $5.28 \mathrm{~cd}$ & $5.98 \mathrm{~d}$ & $18.00 \mathrm{de}$ \\
\hline $\mathbf{T}_{\mathbf{2}} \mathbf{S}_{\mathbf{4}}$ & $75.44 \mathrm{ab}$ & $2.98 \mathrm{ef}$ & $4.67 \mathrm{e}$ & $15.59 \mathrm{fg}$ \\
\hline $\mathbf{T}_{\mathbf{2}} \mathbf{S}_{\mathbf{5}}$ & $73.82 \mathrm{bc}$ & $2.65 \mathrm{ef}$ & $4.05 \mathrm{e}$ & $14.04 \mathrm{gh}$ \\
\hline $\mathbf{T}_{\mathbf{2}} \mathbf{S}_{\mathbf{6}}$ & $74.88 \mathrm{ab}$ & $2.57 \mathrm{ef}$ & $3.07 \mathrm{f}$ & $13.33 \mathrm{hi}$ \\
\hline $\mathbf{T}_{\mathbf{2}} \mathbf{S}_{\mathbf{7}}$ & $77.78 \mathrm{a}$ & $2.03 \mathrm{f}$ & $2.72 \mathrm{~g}$ & $11.78 \mathrm{i}$ \\
\hline $\mathbf{L} \mathbf{S D}$ & 4.04 & 1.45 & 1.30 & 2.01 \\
\hline
\end{tabular}

Table 4. Effect of spermidine application on economic analysis of rice crop at different developmental stages

\begin{tabular}{|c|c|c|c|c|}
\hline Treatments & Expenses (Rs/ha) & Income (Rs/ha) & Net Benefit (Rs/ha) & BCR \\
\hline $\mathrm{T}_{1} \mathrm{~S}_{1}$ & 106307 & 155065 & 48758 & 1.46 \\
\hline $\mathrm{T}_{1} \mathrm{~S}_{2}$ & 128175 & 189764 & 61589 & 1.48 \\
\hline $\mathrm{T}_{1} \mathrm{~S}_{3}$ & 128175 & 195460 & 67285 & 1.52 \\
\hline $\mathrm{T}_{1} \mathrm{~S}_{4}$ & 128175 & 205849 & 77674 & 1.61 \\
\hline $\mathrm{T}_{1} \mathrm{~S}_{5}$ & 150043 & 235409 & 85366 & 1.57 \\
\hline $\mathrm{T}_{1} \mathrm{~S}_{6}$ & 150043 & 237455 & 87412 & 1.58 \\
\hline $\mathrm{T}_{1} \mathrm{~S}_{7}$ & 171911 & 282430 & 110519 & 1.64 \\
\hline $\mathrm{T}_{2} \mathrm{~S}_{1}$ & 136305 & 200514 & 64209 & 1.47 \\
\hline $\mathrm{T}_{2} \mathrm{~S}_{2}$ & 158173 & 217480 & 59307 & 1.37 \\
\hline $\mathrm{T}_{2} \mathrm{~S}_{3}$ & 158173 & 229945 & 71772 & 1.55 \\
\hline $\mathrm{T}_{2} \mathrm{~S}_{4}$ & 158173 & 244884 & 86711 & 1.50 \\
\hline $\mathrm{T}_{2} \mathrm{~S}_{5}$ & 180041 & 270760 & 90719 & 1.52 \\
\hline $\mathrm{T}_{2} \mathrm{~S}_{6}$ & 180041 & 273970 & 93929 & 1.56 \\
\hline $\mathrm{T}_{2} \mathrm{~S}_{7}$ & 201909 & 314019 & 112110 & \\
\hline
\end{tabular}

\section{Discussion}

Water resources are shrinking day by day due to climate change so crop growers, especially the rice growers are evaluating various water saving rice technologies. Aerobic rice is one of attractive way of rice production as farmers can save about $30-40 \%$ irrigation water. But in another way, farmers have also compromise yield too for water saving. We assume that it might be due to decline of growth hormones as plants may feel water stress, so, artificial application of growth regulators may improve the crop strength to couple water stress. Spermidine, is very efficient growth regulator having multiple influence in rice $[3,4]$, so, it can be assessed 
for its role in aerobic rice. We applied spermidin, at various critical growth stages in rice crop, described in detail under materials and method section. Results clearly described that plants improved the crop performance under spermidin application, both in transplanted as well in aerobic rice. Crop yield of aerobic rice was found very near to flooded rice under sperimidin application at Seedling + Tillering + Panicle initiation $\left(\mathrm{S}_{7}\right)$. It might be due to role of spermidin to manage water stress as many metabolic processes are dependent on spermidin [5]. All treatments of spermidin application improved the crop performance as compared with control but it's also obvious from results that its application on reproductive stage, responded more positively. Literature revealed that it promotes vegetative as well as reproductive growth due to activation of different enzymes $[7,8]$. This is the reason, that all yield attributes were improved in spermidin application at three critical stages. [9] also reported that plants having a high concentration of spermidine establish high yield and yield components.

Rice quality has a big importance in the country as its export is highly dependent on good quality. Pakistani rice is very famous all over the world due to its aroma and elongation after cooking. So quality is the major parameter which can't be ignore, whatever we follow cultivation system. Aerobic rice, not only reduce yield but also impair grain quality as per farmer feedback. Our results exhibited that, spermidin application not only improved growth but also resulted in better yield and quality. It might be due to sperimidin ability to work like antioxidant under any stress condition to save plant $[10,11]$, which ultimately saved at growth stages as well which lead toward improved grain quality. Seedling and tillering are two very important growth stages; any stress at these stages may reduce leaf chlorophyll contents which ultimately impair plant photosynthetic efficiency or indirectly the crop yield. Moreover, adoption of any recommendation is highly dependent on economics and it's most important in developing countries like Pakistan. Economics analysis revealed that maximum BCR was achieved in $T_{1} S_{7}$ which explained to apply sperimidin at three different stages in aerobic rice. This analysis has also changed the situation as flooded rice found here inferior due to more cost involved.

\section{Conclusion}

Foliar application of spermidine at three developmental stages; seedling, Tillering and panicle initiation was found to be most appropriate in aerobic rice. Rice producers can maximize their output with spermidine application under limited water resources

\section{Authors' contributions}

Conceived and designed the experiments: $\mathrm{N}$ Sarwar, Performed the experiments: $\mathrm{MH}$ Javaid, A Neelam, Analyzed the data: A Rrhman, U Farooq, Contributed reagents/ materials/ analysis tools: A Ghani, MZ Mushtaq, A Wasaya, Wrote the paper: N Sarwar, K Mubeen.

\section{References}

1. Predeepa JR (2012). Aerobic rice: the next generation innovation in rice cultivation technology. Int J Farm Sci 2: 54-58.

2. Priyanka SB, Jitesh \& Babu S (2012). Aerobic rice, a new approach of rice cultivation. Inter $J$ of Res in Bio Sci 1(1):1-6.

3. Walters DR (2003). Polyamines and plant diseases. Phytochem 64: 97-107.

4. Yuan LP (2008). Hybrid rice breeding for super high yield. Hybrid Rice 12: 14.

5. Shen W, Nada K \& Tachibana S (2000). Involvement of polyamines in the chilling tolerance of cucumber cultivars. Plant Physiol 124: 431-439.

6. Esfandiari E, Shekari F, Shekari F \& Esfandiar M (2007). The effect of salt 
stress on antioxidant enzymes activity and lipid peroxidation on the wheat seedlings. Not Bot Hort Agrobo Cluj 35(1): 48-56.

7. Tan G, Zhang H, Fu J, Wang ZQ, Liu LJ \& Yang JC (2009). Post-anthesis changes in concentration of polyamines in superior and inferior spikelets and their relation with grain filling of super rice. Acta Agro Sin 35: 2225-2233.

8. Liu CM \& Xia J (2004). Water problems and hydrological research in the yellow river and the Huai and Hai river basins of China. Hydro Proc 18: 2197-2210.

9. Feng L, Bouman BAM, Tuong TP, Cabangon RJ, Yanglang L, Guoan L \&
Feng Y (2011). Exploring options to grow rice using less water in northern China. I. Field experiments and model evaluation. Agric Water Manage 88: 113.

10. Alcazar R, Altabella T, Marco F, Bortolotti C, Reymond M, Koncz C, Carrasco P \& Tiburcio AF (2010). Polyamines: Molecules with regulatory functions in plant abiotic stress tolerance. Planta 231: 1237-1249.

11. Zhang HC, Li J, Yao Y, Huo ZY, Dai QG, Xu K, Yang B, Zhao PH \& Wei HY (2009). Scientific Issues of Direct Seeding Rice Cultivation. Chinese Agric Sci Press, Beijing. 